\title{
Optimization of cascaded regenerative links based on phase sensitive amplifiers
}

\author{
M. A. Sorokina,* S. Sygletos, and S. K. Turitsyn \\ Aston Institute of Photonic Technologies, Aston University, B4 7ET Birmingham, UK \\ *Corresponding author: sorokina@aston.ac.uk \\ Received June 21, 2013; revised August 31, 2013; accepted September 19, 2013; \\ posted September 19, 2013 (Doc. ID 192684); published October 24, 2013
}

\begin{abstract}
We develop an analytical method for optimizing phase sensitive amplifiers for regeneration in multilevel phase encoded transmission systems. The model accurately predicts the optimum transfer function characteristics and identifies operating tolerances for different signal constellations and transmission scenarios. The results demonstrate the scalability of the scheme and show the significance of having simultaneous optimization of the transfer function and the signal alphabet. The model is general and can be applied to any regenerative system. C 2013 Optical Society of America

OCIS codes: (060.2330) Fiber optics communications; (060.4370) Nonlinear optics, fibers; (190.4370) Nonlinear optics, fibers; (060.1660) Coherent communications; (000.6800) Theoretical physics.

http://dx.doi.org/10.1364/OL.38.004378
\end{abstract}

The increasing capacity demand in communication systems has introduced signal technologies of high constellation complexity to achieve a better exploitation of the available bandwidth [1]. As the trade-offs between constellation size and transmission reach are strict [2], frequent regeneration functionality will be unavoidable in future fiber links. All-optical regeneration represents a unique low-cost and energy-efficient solution for improving the transparent length and enabling high-capacity networking.

Future all-optical regenerators will have to deal with advanced phase encoded signal formats and enable direct removal of phase distortions [3]]. Phase sensitive amplifiers (PSA) have recently appeared as an ideal platform to support this functionality, and subsystems for binary [3] and higher-order phase encoded formats have already been demonstrated [4]. Although these schemes have been designed and characterized for single stage demonstrations, their cascadability performance remains unknown. A rigorous optimization of their regenerative transfer function is necessary to achieve effective suppression of the transmission impairments in cascaded systems. A first approach has been proposed in [5], which is based on a "misfit factor" to minimize the difference between the nonlinear response of the PSA and the stepwise transfer function of an ideal multilevel quantizer. Recently, in [6], an analytical methodology has been developed to optimize the transfer function of nonlinear regenerative systems based on the well-known theory of dynamic system analysis [7]. This enables us to make analytical predictions for optimal parameters.

In this Letter, we apply the analytical approach of [6] to design PSA-based regenerator cascades for multilevel phase encoded signal transmission. Our method is focused on the optimization of the transfer function that is responsible for the phase noise squeezing capabilities of the regenerator and affects its overall nonlinear response. In particular, through a set of simple mathematical conditions, we define the optimal signal alphabet and identify the operating margins of the PSA cascade. The analytical predictions of our theory are verified by rigorous numerical simulations of the transmission link performance. We show that in cascades the sensitivity to optimization increases and the derived analytical expression for the optimal PSA parameter ensures the best performance. The proposed model is generic and can be applied to any regenerative transfer function providing the possibility of dramatic improvements in the information capacity of future transmission systems [].

An M-level all-optical phase quantizer can be realized by means of a PSA scheme that enables coherent addition of an input signal $r e^{i \varphi}$ and its $(M-1)$ order phase conjugated harmonic according to

$$
R e^{i \psi}=r e^{i \varphi}\left(1+m e^{-i \varphi M}\right) .
$$

Here, $R$ is the amplitude response of the regenerator, characterized by a sinusoidal dependence on the phase of the input signal, and $\psi=F(\varphi)$ is its staircase phase response, which can be written as

$$
\psi=F(\varphi)=\tan ^{-1}\left(\frac{\sin [\varphi]+m \sin [\varphi(1-M)]}{\cos [\varphi]+m \cos [\varphi(1-M)]}\right) .
$$

In the above equations $m$ corresponds to the amplitude ratio of the interfering signal-idler pair of waves. It is an optimization parameter that defines the slope of the phase transfer function of the PSA near the alphabet points and characterizes its regenerative properties (see Fig. 1). More specifically, the phase transfer function creates a periodic attractive potential around a set of special stationary points that are unaffected by the nonlinear transformation. Both the stationary points and the attractive potential contribute to the phase squeezing performance of the PSA. Clearly, the optimum set of $m$ parameters has to be identified to maximize the regeneration efficiency. Since the regenerative channel is discrete, it is also necessary to adapt the characteristics of the nonlinear element to the employed signal format and vice versa.

First, the nonlinear transfer function of the regenerator is adjusted to the signal alphabet. For this, the attraction regions should be centered at the corresponding alphabet points-the stationary points of the transformation, 


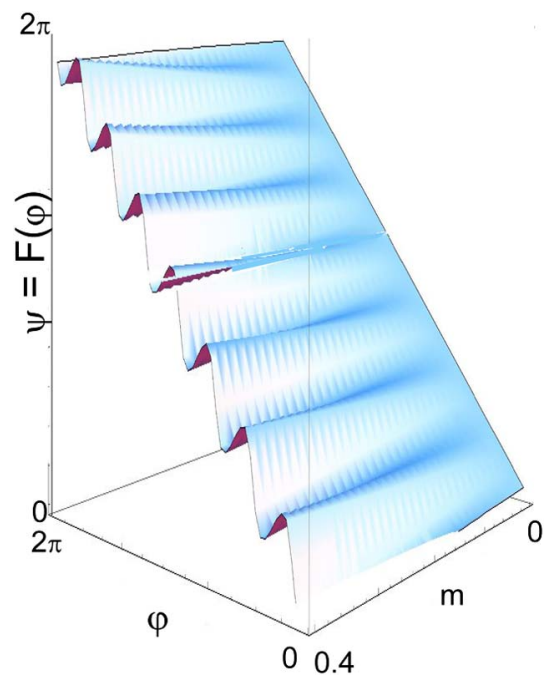

Fig. 1. Periodicity and slope characteristics of the PSA phase transfer function with varying input phase $\varphi$ and regenerative parameter $m$.

as defined by the conditions $F^{\prime \prime}\left(\varphi^{*}\right)=0$ and $F\left(\varphi^{*}\right)=\varphi^{*}$. Thus, starting from Eq. (2), we derive the optimum constellation given by the set $\varphi^{*}=l \pi / M, l \in Z$.

The next step is to ensure the stability of the nonlinear transformation at the alphabet points for effective suppression of the phase noise. This condition leads to the inequality $\left|F^{\prime}\left(\varphi^{*}\right)\right|<1$, from which the variation limits of the regenerative parameter $m$ are derived as

$$
|m|<m_{c r}, \quad m_{c r}=\frac{2}{M-2} .
$$

Here $m>0$ if $l=2 k$ and $m<0$ if $l=2 k+1$, with $k \in Z$. The points are superstable, i.e., $F^{\prime}\left(\varphi^{*}\right)=0$, when

$$
\left|m_{\mathrm{opt}}\right|=\frac{1}{M-1} .
$$

The superstable case creates operational plateaus around the alphabet points of the transfer function that enable maximum phase noise suppression. For $m \neq m_{\text {opt }}$ plateaus cannot be defined; however, provided that $|m|<m_{c r}$, partial suppression of the phase noise is still feasible within the limits of each attraction region, since $\left|F^{\prime}\left(\varphi^{*}\right)\right|<1$. An analytical expression can be derived for the attraction region's half-width $\Delta$ in the limit of $\Delta \ll 1$ by performing perturbation analysis on the equation $\left|F^{\prime}\left(\varphi^{*}+\Delta\right)\right|=1$, resulting in

$$
\Delta=\sqrt{\left(1-\left|\frac{-1-m+m M}{1+m}\right|\right) /\left(\frac{m M^{3}(1-m)}{2(1+m)^{3}}\right)} .
$$

The parameter $\Delta$ has the meaning of the maximum phase noise distortion that can be suppressed by the regenerator. It acquires the maximal value $\Delta_{\max }$ for the plateau condition $m=m_{\text {opt }}$, whereas for suboptimal $m$ values it narrows and tends to zero when $m$ approaches $m_{c r}$.

Figure 2(a) depicts the phase transfer functions of regenerative PSAs with eight discrete phase states. The green solid line corresponds to the $m_{\mathrm{opt}}$ selection of the regenerative parameter. The condition, $F^{\prime}\left(\varphi^{*}\right)=0$, leads to a joint optimization of the amplitude and phase response of the PSA, as it achieves simultaneous suppression of its phase-to-phase and phase-to-amplitude noise conversion mechanisms. The red dashed line has been taken for the critical value $m_{c r}$, above which the PSA elements amplify phase noise and degrade the system performance. Figure 2(b) shows the dependence of the optimal and critical values of the regenerative parameter $m$ on the constellation size $M$. One can see that for highorder modulation formats the gap between these two values is narrowed, making the PSA optimization more critical. The variation of the corresponding half-width $\Delta_{\max }$ is also depicted in the same figure.

Subsequently, the accuracy of the proposed analytical optimization method is verified by simulating numerically the performance of quasi-linear transmission regimes with cascaded PSA-based regenerators. The PSAs have identical transfer functions and are placed equidistantly along the transmission line. The transmission impairments are represented by additive white Gaussian noise (AWGN) distributed uniformly along the line. The introduced degradation has been quantified in terms of the signal-to-noise ratio (SNR), defined as the ratio of the input signal power to the power of the linearly accumulated noise along the transmission link, i.e., in the absence of PSA regenerators. This convention provides a common reference for the benchmarking of different nonlinear transmission channels [8]. The system performance has been characterized in terms of symbol error rate (SER) calculated by direct error counting of $2^{23}$ Monte Carlo generated symbols. By comparing the SER while varying system parameters, we analyzed the general trends and evaluated the performance gain due to the regenerative functionality in the transmission system.

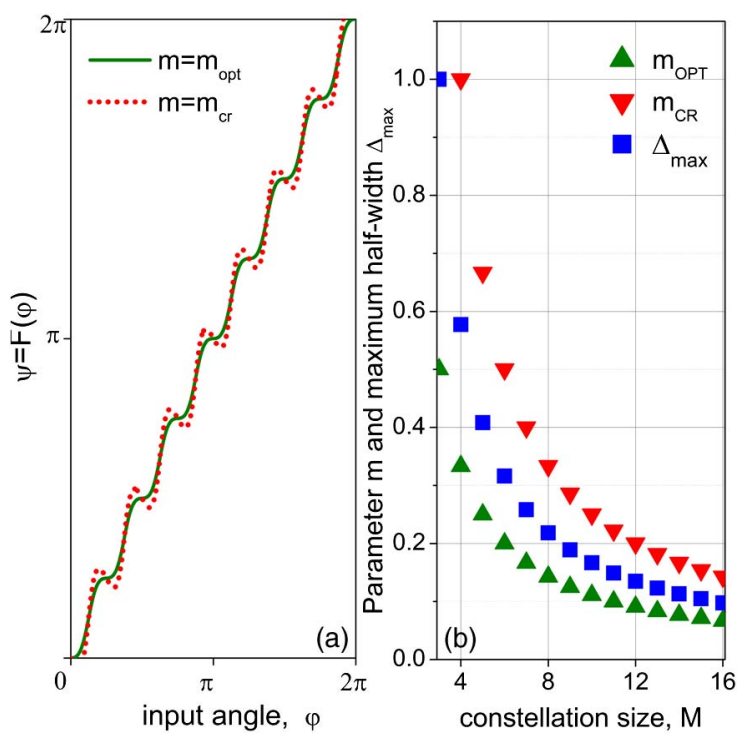

Fig. 2. (a) PSA phase transfer function for 8-PSK format. The optimal value of the parameter $m_{\text {opt }}=1 / 7$ (shown by green solid line) demonstrates a plateau centered at the alphabet points, whereas a critical choice $m_{c r}=0.33$ of the parameter (shown by red dashed line) is expected to lead to poorer regenerative performance. (b) Dependence of the optimal and critical values of $m$ and the attraction region's maximum half-width $\Delta_{\max }$ on the order $M$ of the PSK modulation format. 


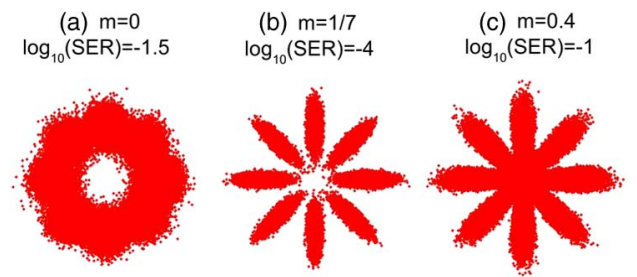

Fig. 3. Constellation diagram of 8-PSK after transmission with $\mathrm{SNR}=12 \mathrm{~dB}$ (a) without regeneration and after 10 PSAs in cascade with (b) optimal $m=1 / 7$ and (c) nonoptimal $m=0.4$. One can observe dramatic gain in system performance due to the PSA optimization for the cascaded scenario.

Constellation diagrams of an 8-PSK signal after propagation through a transmission line of 10 cascaded PSAs at $\mathrm{SNR}=12 \mathrm{~dB}$ are depicted in Fig. 3. The results have been taken for different selections of the parameter $m$, highlighting the influence of the PSA transfer function on the quality of the propagated signal. The unregenerated case is depicted in Fig. 3(a). It is clear that a significant SER improvement occurs for $m=m_{\text {opt }}$ [see Fig. 3(b)] when the PSA achieves efficient suppression of the induced phase noise. At the same time, a slight increase of the amplitude variation is noticed, as the gain of the PSA is also phase dependent. For $m \geq m_{c r}$ [see Fig. 3(c)], the phase distortion at each PSA stage is enhanced, leading to a worse system SER performance than the unregenerated case. However, the constellation shape of the signal maintains the basic star-like features of the regenerated case (middle panel) due to the sinusoidal amplitude response.

Figure 4 depicts the SER performance as a function of the SNR for various numbers of cascaded PSAs and for 4, 8, and 16-PSK modulation formats. For comparison, the results are presented in two sets: the optimum and nonoptimal values of the $m$ parameter. For the nonoptimal selection of $m$, a rapid degradation of the system performance occurs. On the contrary, when $m=m_{\text {opt }}$, the strongly regenerative behavior of the PSAs leads

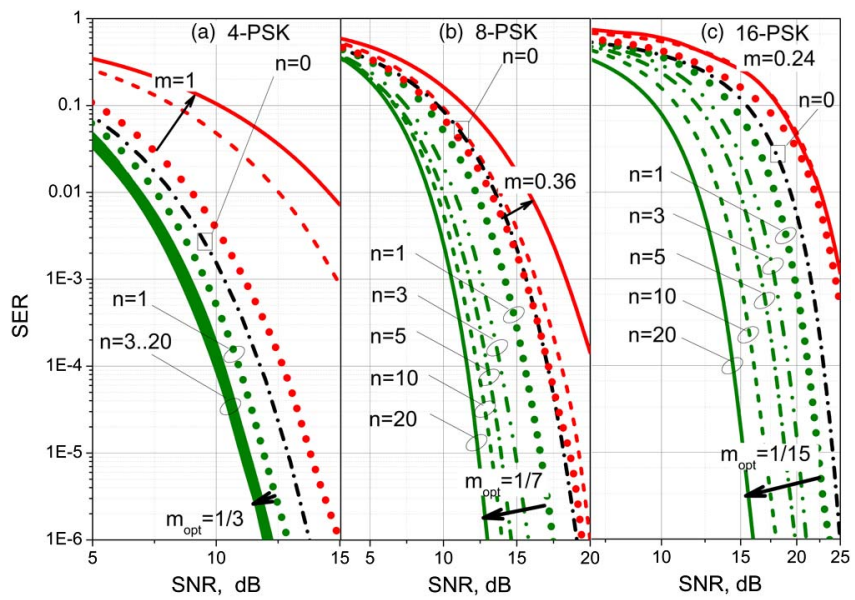

Fig. 4. SER as a function of SNR with cascaded regenerative PSA elements for (a) 4-PSK, (b) 8-PSK, and (c) 16-PSK formats. The SER of the linear channel is shown for comparison with a black dashed-dotted line. The SER of the system with the optimal and critical values of the parameter $m$ is shown with green and red curves, respectively. The arrows show the SER evolution for increasing number of inline PSAs. to a significant performance enhancement along the cascade, which is more profound for higher-order modulation formats. Indeed, at SER of $10^{-3}$ the SNR improvement, with respect to the unregenerated (linear) system, is only $1.5 \mathrm{~dB}$ for the QPSK signals, whereas for the 8 and 16-PSK signals it is 5.2 and $8 \mathrm{~dB}$, respectively. The results underline the increasing importance of all-optical regeneration with the constellation size of the transmitted signal. Specifically, they suggest that future PSA implementations should target modulation formats of higher order than QPSK, for nonincremental improvements in system performance. As the number of cascaded PSAs increases, asymptotic behavior in performance improvement is also observed; see Fig. $\underline{4}$. This occurs because the transmission line is divided to a large number of sections and the noise power generated by each of them becomes significantly low to create phase errors. For higher-order modulation formats, it takes a larger number of inline regenerators to reach this saturation point as the signal sensitivity to phase noise is also increased.

We have investigated further the performance dynamics along the PSA cascade by calculating the corresponding SNR improvement (for fixed SER $=10^{-3}$ ) as a function of the regenerative parameter $m$. Figure 5 depicts results for the case of an 8-PSK signal and for a different number of inline regenerators. The aforementioned saturation effect in the performance is more apparent in this figure. Increasing the number of inline regenerators from $n=1$ to $n=2$, gives the same SNR gain of $\sim 1 \mathrm{~dB}$ as we get from $n=10$ to $n=20$. The dependence of the phenomenon on the regenerative parameter $m$ is also shown. The saturation occurs faster for suboptimal $m$ values. This is attributed to the nonideal transfer function of the PSA, which allows an amount of residual phase distortion to accumulate

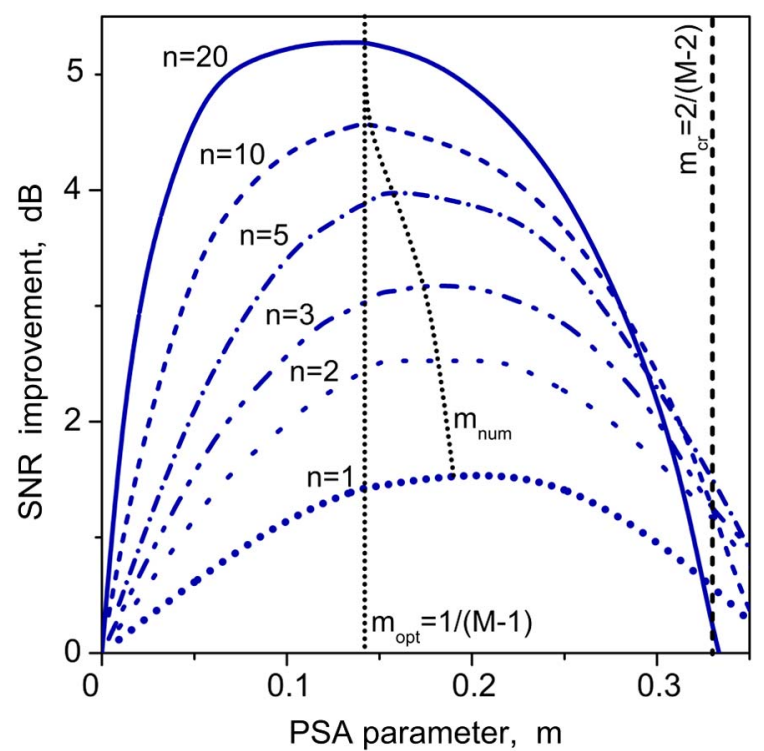

Fig. 5. Dependence of the SNR improvement due to PSA application for the fixed value SER $=10^{-3}$ as a function of the PSA parameter $m$ for 8-PSK modulation format for the different numbers of PSAs (denoted by $n$ ). Faster saturation in performance improvement occurs for nonoptimal $m$ values. The convergence of the numerical values $m_{\text {num }}$ to the analytical $m_{\text {opt }}$ is shown by a black dotted curve. 


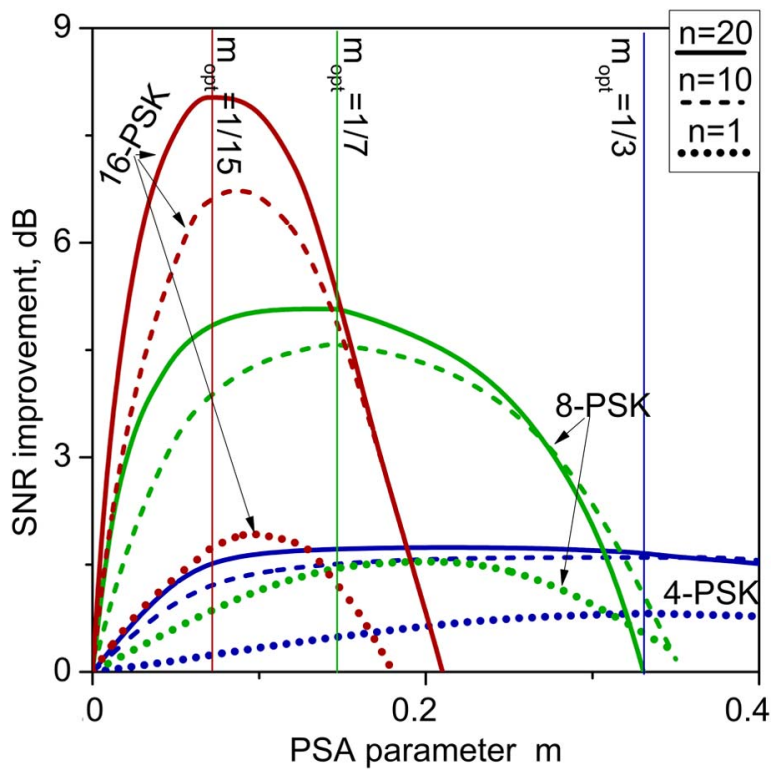

Fig. 6. Dependence of the SNR improvement due to PSA application for the fixed value SER $=10^{-3}$ as a function of the PSA parameter $m$ for 4 (blue), 8 (green), and 16 (red) PSK modulation formats. The cascaded scheme of 10 (dashed) and 20 (dotted) PSA elements gives significant improvement for high-order PSK. The sensitivity to the optimization parameter $m$ increases rapidly for dense formats. The optimal value of $m$ is shown by vertical lines of the corresponding color for each constellation size.

between the regenerative sections enhancing the overall SER degradation. In Fig. $\underline{5}$, we have also identified the analytically predicted $m_{\text {opt }}$ values along with the corresponding optima indicated by the numerical simulations. A slight deviation is apparent between them for a small number of cascaded PSAs. However, since at this regime the system performance is relatively insensitive to $m$, the corresponding impact in SNR improvement is minimal, i.e., less than $4 \%$.

Figure 6 extends the results of the previous figure to different modulation formats (i.e., 4, 8, and 16-PSK). We may observe that a plateau in the transfer function of the regenerator is not a necessary condition for demonstrating phase regeneration along the cascade. It is also possible to achieve effective noise suppression for suboptimal choices of the $m$ parameter. For example, selecting a suboptimal value $m=0.3$ gives, for the case of 8-PSK signals, an SNR improvement of $2 \mathrm{~dB}$, whereas the corresponding transfer function does not show any plateau-like behavior. On the other hand, nonoptimal $m=0.36$ provides a similar transfer function, but it degrades the system performance compared to the channel in the absence of PSA, as it slightly exceeds the critical value of $m=0.33$, which our theory has predicted.

We have proposed an analytical technique to define, in terms of single transfer function parameter $m$, the optimum conditions and operating margins of cascaded PSA-based regenerators in multilevel phase encoded transmission links. Rigorous numerical simulations have been carried out and have confirmed the analytical predictions of our theory. Furthermore, they have revealed the performance dynamics along the cascaded system. It has been found that all-optical phase regeneration brings significant performance gain to the system, when moving to larger signal complexities, but reduces the operating margins. A saturation effect in performance improvement has also been predicted, which evolves more rapidly for nonoptimal transfer functions and high modulation orders. The developed theory will be essential for the design of future high-capacity transmission systems with all-optical regenerators.

Prof. A. D. Ellis is gratefully acknowledged for useful discussions. This work has been supported by the EPSRC project UNLOC (Unlocking the capacity of optical communications) EP/J017582/1, grant of the Russian Ministry of Education and Science Federation (agreement N11.519.11.4001), and the Marie Currie-IEF grant ARTISTE.

\section{References}

1. P. J. Winzer and R.-J. Essiambre, J. Lightwave Technol. 24, 4711 (2006).

2. R. J. Essiambre, G. Kramer, P. J. Winzer, G. J. Foschini, and B. Goebel, J. Lightwave Technol. 28, 662 (2010).

3. R. Slavik, F. Parmigiani, J. Kakande, C. Lundström, M. Sjödin, P. A. Andrekson, R. Weerasuriya, S. Sygletos, A. D. Ellis, L. Grüner-Nielsen, D. Jakobsen, S. Herstrøm, R. Phelan, J. O'Gorman, A. Bogris, D. Syvridis, S. Dasgupta, P. Petropoulos, and D. J. Richardson, Nat. Photonics 4 690 (2010).

4. J. Kakande, R. Slavík, F. Parmigiani, A. Bogris, D. Syvridis, L. Grüner-Nielsen, R. Phelan, P. Petropoulos, and D. J. Richardson, Nat. Photonics 5, 748 (2011).

5. J. Kakande, R. Slavik, F. Parmigiani, P. Petropoulos, and D. Richardson, in Optical Fiber Communication Conference (Optical Society of America, 2012), p. OW1I.3.

6. M. A. Sorokina and S. K. Turitsyn, http://arxiv.org/abs/1305 .1537 .

7. R. M. May, Nature 261, 459 (1976).

8. K. S. Turitsyn and S. K. Turitsyn, Opt. Lett. 37, 3600 (2012). 